\title{
Vamos a construir paz todos: unidad didáctica para la reflexión acerca de la construcción social y cotidiana de la paz ${ }^{1}$ \\ Let's all build peace: Didactic unit for the reflection about the social and quotidian construction of peace
}

\author{
Alison Lorena Fuentes Oliveros* \\ Fernanda Carolina Arévalo Olaya**
}

\section{Resumen}

En este artículo presentamos el proceso de construcción de la unidad didáctica Vamos a construir paz todos, fruto del trabajo de grado para obtener el título de Trabajadoras sociales. Optamos por el paradigma cualitativo y enfoque de educación popular en clave decolonial, como ejercicio reflexivo y práctico para trascender el ámbito académico y proyectar reflexiones acerca del papel que todos tenemos en la construcción social de la paz, proceso que integramos con nuestras prácticas cotidianas y nos compete como ciudadanos. Ubicamos los conceptos de construcción social de paz, cotidianidad, diálogo de saberes y subjetividad en la educación popular, la pedagogía y la didáctica. Como fruto del proceso investigativo, creamos una unidad didáctica que a través de tres mediaciones lúdicas, aporta premisas para la reflexión acerca de la construcción social de la paz.

Palabras clave: Construcción de paz; Diálogo de saberes; Educación popular; Cotidianidad; Unidad didáctica.

${ }^{1}$ El artículo es fruto de reflexiones grupales en el marco del proyecto de grado Vamos a construir paz todos: unidad didáctica para la reflexión acerca de la construcción social y cotidiana de la paz presentado en la Universidad de La Salle, Colombia, en el año 2015. Escribimos el texto en forma personal y plural: realizamos la reflexión Carolina Arévalo y Alison Fuentes, fruto de experiencia grupal en que participaron también Magaly Robayo y Alejandra Hernández, hoy colegas, con quienes compartimos el proceso académico. Se trata de una escritura desde el corazón y con el fin de continuar aportando a la construcción social y cotidiana de la paz.

*Trabajadora social Universidad de la Salle. Correo electrónico: alis.28.03@gmail.com

*** Trabajadora social Universidad de la Salle. Correo electrónico: caroarevalo1@gmail.com

Recibido: 30 de Abril de 2017 Aprobado: 28 de junio 2017

ISSN: 0122-1213 (Impreso) ISSN: 2389-993X (En línea) Doi: 10.25100/prts.v\%vi\%i.4544 


\section{Abstract}

In this article we show the process of building the didactic unit "Vamos a construir paz todos", which is the result of the final project required to aspire to a degree of a social worker. We choose a qualitative paradigm and a context of popular education in decolonial way approach, as a reflective and practical exercise that seeks to transcend academic discussion and generate reflections about the role we all have in the social construction of peace; this because it is a process integrated in our daily practices and concern us as citizens. Our discussion encompasses the concepts of social construction of peace, daily life, dialogue of knowledge, subjectivity in popular education, pedagogy and didactics. As a result of the investigative process, we create a didactic unit that provides, through three playful mediations, premises for the reflection about the social construction of peace.

Key words: Peace building; Dialogue of knowledge; Popular education; Daily life; Didactic unit.

Sumario: 1. Introducción, 2. Perspectiva teórica-conceptual, 3. Metodología, 4. Resultados, 5. Conclusiones, 6. Referencias bibliográficas

\section{Introducción}

Paz es una palabra que los colombianos escuchamos con regularidad, especialmente en estos últimos años, con la coyuntura del proceso de paz, motivado por el gobierno Santos. Sin embargo, no hay que ser presidente para darse cuenta que la paz es necesariamente una construcción social que debe pasar por la vida cotidiana de cada ciudadano; que claramente debe atravesar los sistemas estructurales y los conflictos políticos que permean la historia del país para trascender a la construcción de una paz cultural y permanente. Al hacer una revisión de diferentes iniciativas de paz, encontramos muestras artísticas, organizativas, pedagógicas y movilizadoras de diferentes comunidades; también intentos de acuerdos con los actores armados, leyes, programas y políticas parciales e inefectivas en la práctica. Más allá de "qué hubo", lo cierto es que son esfuerzos que Prospectiva. Revista de Trabajo Social e Intervención social No. 24, julio-diciembre 2017: pp. 177-203 
incluyen a pequeñas partes de la población colombiana, es decir, que la gente que llamamos "del común", muchas veces no conoce qué se ha hecho para alcanzar la paz, tampoco siente que sus acciones, sus vidas y sus reflexiones sean un potencial aporte para la construcción de la misma; por el contrario, la sienten "por fuera de ellas".

Por esta razón, en el año 2015 desarrollamos el trabajo de grado "Vamos a construir paz TODOS. Unidad didáctica para la reflexión acerca de la construcción social y cotidiana de la paz". Buscábamos trascender el ámbito académico, llegar al corazón, a la cotidianidad de las personas, con reflexiones pedagógicas y lúdicas, que nos permitieran entender que todos hacemos parte del proceso de construcción de paz.

En las opciones de trabajo de grado en el programa de Trabajo Social de la universidad de La Salle, se contempla la modalidad de "Creación de materiales de apoyo para procesos de intervención". La investigación y producción de la unidad se enmarca en la línea de investigación Desarrollo Humano y Calidad de Vida, la sub-línea de Justicia Social, que:

Permite hacer una comprensión acerca de las realidades y ubicar los debates sobre paz en el marco de la cotidianidad y los principales problemas sociales, entendiendo la alteridad de los escenarios y la necesidad de fortalecer procesos que construyan caminos para la vivencia de la paz social y cuestionen las estructuras (Arévalo, Fuentes, Hernández y Robayo, 2015, p 20).

\section{Perspectiva teórica-conceptual}

En el reto de crear un material que permitiera cumplir con el objetivo de generar reflexiones cotidianas frente a la construcción de paz, la argumentación epistemológica, teórica y conceptual fue fundamental: retomamos la epistemología decolonial revisando a Mignolo (2007), Quijano (1992) y Marañon (2016) y la educación popular con Freire (1964, 1966), Peresson (1983); Alfonso Torres (2000), Dussán (2004), perspectivas que juntas permitieron entender la educación popular en clave decolonial, y nos llevaron a profundizar en los conceptos de construcción 
de paz, diálogo de saberes, cotidianidad y prácticas cotidianas, subjetividad en la educación popular, pedagogía y didáctica (Véase Figura 1).

Figura 1. Espiral de conceptos que fundamentan la investigación

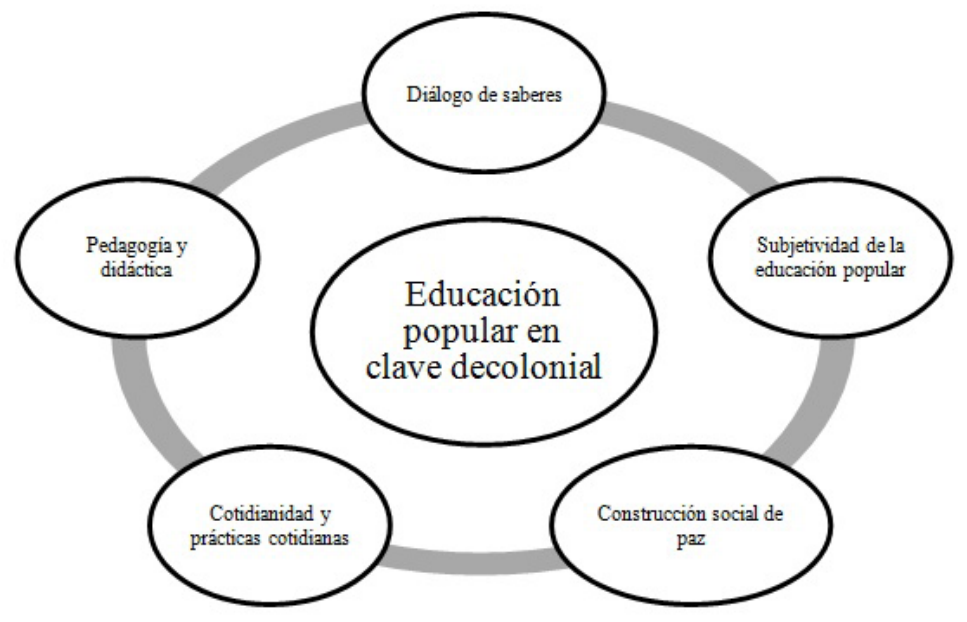

Fuente: Elaboración propia

Al revisar la documentación sobre epistemología decolonial, nos encontramos con una perspectiva teórica que implica la decolonización de los saberes, es decir, transformar las ideas hegemónicas impuestas por la colonización -los modelos europeos occidentales normalizados y legitimados- para construir conocimientos propios que respondan a las dinámicas históricas, sociales y económicas de cada contexto. Mignolo (2007) plantea en el giro decolonial, esta perspectiva como:

Una consecuencia de la formación y ejecución hegemónica de poder en los diferentes territorios, teniendo como razón de ser y objetivo principal la decolonización del conocimiento. Se refiere a un nuevo diálogo intercultural, un intercambio de saberes, significados y experiencias de las diferentes culturas (citado por Arévalo, Fuentes, Hernández y Robayo, 2015). 
La decolonialidad, según Quijano (1992), nace en el mismo momento que la colonialidad como su opuesto, sin embargo, el estudio de la misma ha sido objeto de un debate contemporáneo que ha sido protagonizado especialmente por países latinoamericanos quienes han sido deslegitimados por las ideas eurocéntricas, o en palabras de De Sousa (2010), por el sur no geográfico.

La colonialidad se refiere al proceso de organización social a través de la raza mediante una estructura jerárquica que otorga características y habilidades de acuerdo con el nivel social ocupado que es asignado con base en "supuestas diferencias biológicas" que nos hacen desiguales en oportunidades; es decir, la colonialidad excluye y vuelve objeto todo lo diferente a "blanco, hombre, europeo, burgués y heterosexual", lo que se salga de dichos parámetros es pormenorizado y juzgado para que no sea tenido en cuenta.

El éxito de la colonialidad surge con la llegada de la modernidad, cuando las diferentes sociedades asumieron el discurso del desarrollo como una manera de ingresar en la estructura de poder, por medio de organismos y tratados internacionales; pero también invisibilizando las culturas y cosmovisiones propias de los países para entrar en la idea homogeneizante de la globalización, la libertad y autonomía humana sobre la naturaleza.

La decolonialidad es entonces la antítesis de la colonialidad, que como corriente epistemológica ubica la necesidad de retornar a las ideas ancestrales de los pueblos, la reivindicación de los explotados, el reconocimiento de los saberes propios, la creación de conocimientos contextualizados, el respeto por la naturaleza, la potenciación de la relación con el otro y la diferencia como forma de socialización y fin último para la construcción de sociedades con una racionalización diferente dirigida al buen vivir (Marañon, 2016).

De igual forma, la decolonialidad debe ser entendida como la forma de reivindicación social de "lo no europeo" en todas sus dimensiones: trabajo-economía, sexualidad, naturaleza, Estado-política, Subjetividad y conocimiento; por ello, encontramos en esta perspectiva una sombrilla que nos permite comprender la realidad de una manera diferente y crítica 
para la construcción de una sociedad sin exclusión, con justicia social, reciprocidad y solidaridad.

Según Torres (2000), en la educación popular encontramos una corriente político-epistemológica en que se comprende el proceso educativo como práctica misma de la libertad (Freire, 1964), en la que se plantean relaciones dialógicas y recíprocas que propician la construcción de conocimiento desde las experiencias y vivencias de cada sujeto. Peresson (1983) citado por Dussán (2004), afirma que la educación popular es una corriente propuesta desde los sectores sometidos y silenciados, como una manera de generar sujetos históricos y protagonistas de los cambios y transformaciones sociales que buscan en sus comunidades.

De esta forma, comprendimos que la educación popular en clave decolonial implica una visión crítica de la historia, en la que se buscan prácticas educativas que permitan a los sujetos emanciparse de las ideas hegemónicas y ser parte de los procesos de transformación social.

La educación popular en perspectiva decolonial apuesta a metodologías alternativas que integran el saber con el hacer y el sentir con el pensar: procesos didácticos que fortalezcan la participación y la reflexión social (Arévalo, Fuentes, Hernández y Robayo, 2015, p. 38).

Desde la educación popular en clave decolonial como eje epistemológico, buscamos desarrollar los conceptos de construcción de paz, cotidianidad, prácticas cotidianas y diálogo de saberes. Profundizamos también sobre la dimensión subjetiva de la educación popular, la pedagogía y la didáctica, formando una "espiral de conceptos" que representamos en el gráfico 1.

Para entender la construcción social de paz, retomamos aportes de Galtung (2003), la Escola de Cultura de Pau (2011), García-Durán (2006), Bautista (2013) y Fontan (2013), quienes permiten comprender procesos en construcción, situados, con múltiples maneras de entender, que en el contexto colombiano la paz tiene maneras específicas de vivirse y soñarse, luego, presentan varias concepciones de paz que analizamos bajo la lupa de la realidad histórica del país.

Galtung (2003) retoma la idea dicotómica de paz negativa y paz positiva, la primera caracterizada por la ausencia de guerra, la supresión o carencia 
de los conflictos armados o el desarme, y la segunda, suponiendo un nivel mínimo de violencia directa y elevado nivel de justicia, no es lo contrario de la guerra, sino la ausencia de violencia estructural, aquella que forma parte de la estructura social y que impide cubrir las necesidades básicas, como las generadas por la desigualdad social: la malnutrición, la carencia de servicios sanitarios y educativos básicos; y se centra en la armonía del ser humano consigo mismo, con los demás y con la naturaleza, además, no supone un rechazo de los conflictos, al contrario, expone que es necesario aprender a afrontarlos y resolverlos de forma pacífica y justa. Esto implica que existen situaciones de tipo estructural que generan condiciones de violencia, puesto que no se garantizan escenarios justos para el desarrollo de la vida de las personas. Bautista (2013) expresa que se debe hacer un análisis de la paz, desde una concepción de solución política; esto es, entender que hay diversos sectores en la sociedad que entienden el alcance de la paz desde diferentes concepciones. Existe una idea de que la paz existe solo con la derrota total de los sectores que generan amenaza social, o terroristas; en otra, se comprende que el Estado y los sectores dominantes tienen la capacidad de mitigar los conflictos y las contradicciones, reconociendo que existen situaciones de desigualdad, inequidad y exclusión generadas por el modelo económico basado en el mercado; y una tercera, en la que se identifica que el desarme y la generación de acuerdos políticos, sociales y económicos, generan transformaciones estructurales a los problemas que sostienen la existencia del conflicto armado y la injusticia social (Bautista, 2013).

La Escola de Cultura de Pau (2011) entiende la paz como el conjunto de acciones y esfuerzos en que se busca la transformación de escenarios violentos en relaciones pacíficas y sostenibles, independientemente del momento en el que se adelanten. Es decir, que dichas acciones se pueden adelantar antes, durante o después del conflicto armado; además, propone una concepción del conflicto como oportunidades para dar cabida al cambio, la adaptación, la comunicación y el intercambio.

En este sentido, García Durán (2006) habla de dos posturas contradictorias: una que busca por medios violentos alcanzar la paz, y otra que busca medios no violentos para dicho fin; propone reflexionar 
alrededor de si se debe parar la guerra para construir país o si se construye país para parar la guerra (Gárcía-Durán, 2006, p. 265). Igualmente, plantea caminos como la educación, la investigación, la creación de alternativas políticas y la movilización social para alcanzar la paz.

Victoria C. Fontan (2013) manifiesta la importancia de realizar críticas a la llamada paz liberal, cuestionando las fragmentaciones, inconsistencias y prioridades que gobiernan los ambientes de posconflicto, plantea la necesidad de evaluar la importancia o el valor de los individuos sobre otros y hace referencia a sujetos que se benefician de una paz cuya expresión es meramente industrial e idealista. Es por ello que es inminente hablar de la decolonización de la paz, la cual implica una introspección de todos los aspectos en la industria de la paz, la trascendencia de la élite estructural hacia la formación y facilitación de procesos de paz endógenos, comunitarios y sostenibles (Fontan, 2013, pp. 30-37).

La paz desde la perspectiva decolonial, implica un cambio de racionalidad que requiere del reconocimiento del otro, de las diferencias como una potencialidad para la construcción de proyectos colectivos; por tanto, también invita a retomar las cosmovisiones ancestrales, a resignificar las relaciones sociales desde el diálogo intercultural y con la naturaleza, con todo lo vivo que nos rodea. Ese cambio de racionalidad es tanto personal, como colectivo; es decir, implica condiciones estructurales que no dividan a la sociedad ni la jerarquicen, en donde todos tengamos iguales oportunidades para la garantía del buen vivir de los ciudadanos, con su heterogeneidad; busca, además, un sistema político transparente, construido a partir de la participación activa y consciente, con base en las necesidades, sueños y contextos de las comunidades locales y sus territorios.

De esta manera, construimos una aproximación al concepto de paz, en la que se retoma la dimensión cultural de manera dinámica, recalcando la importancia de pensar y construir paz desde las expresiones y vinculaciones de las comunidades, en las que a partir de experiencias y aprendizajes reflexivos, se transite hacia la transformación en las formas de relacionamiento con los otros y con la naturaleza, potenciando los recuerdos y la memoria histórica, como parte del camino hacia la construcción de paz 
sostenible en el tiempo (Escola de Cultura de Pau, 2011). Entendimos la paz como:

(...) un conjunto de diferentes apuestas que permiten un estado de armonía prolongado en las diferentes dimensiones del sujeto. La paz pasa por la ausencia de violencia, pero también por la justicia social, la convivencia y la resolución pacífica de conflictos, por ello, es un eje transversal en las relaciones sociales, inter e intra personales. Lo anterior nos permite entender que la paz es un proceso, por ende no es un fin en sí misma, es una construcción social a la que debemos contribuir desde nuestra participación con una ciudadanía activa y a la que podemos aportar desde la cotidianidad como sujetos individuales, familiares y colectivos; es decir, desde nuestro actuar personal, en nuestras familias, con los otros sujetos como amigos, compañeros, jefes, incluso, personas que no conocemos; y como ciudadanas y ciudadanos, en nuestra actitud crítica, participativa e interesada por el mundo que nos rodea (Arévalo, Fuentes, Hernández y Robayo, 2015, p. 34-35).

Al referirnos a la cotidianidad, nos fundamentamos en los aportes conceptuales de Heller (1987) y Gómez, De Pablos y Pascual (1999); quienes reconocen lo cotidiano como el foco en el que se reproducen y mantienen las rutinas de receptividad y socialización. Sin embargo, es un espacio que no permite una categorización racional, dado que ocurre en “el ahora”, luego, es espontáneo. Mackay (1997) retomado por Gómez, De Pablos y Pascual (1999), presenta tres concepciones para comprender la vida cotidiana: habla del lugar antropológico en el que se tienen en cuenta los ciclos secuenciales entre lo excepcional, lo rutinario, lo sagrado y lo profano. De otro lado, un sentido político; la ve como lo opuesto al sistema social, es decir lo espontáneo, irracional, lo no planeado que surge de la comunidad y sus emociones. Finalmente, la vida cotidiana como el espacio de la configuración de identidad y de construcción, internalización y socialización de representaciones sociales.

Por su parte, Heller (1987) destaca la particularidad de lo cotidiano, es decir, la manera como cada hombre -y mujer- apropian la vivencia de la cotidianidad, de diferente forma. Comprende que el concepto no hace referencia únicamente al "ahora" o el "hoy", sino que tiene historia y 
futuro, en tanto es cada "hombre -y mujer- particular", el que permite la reproducción de las dinámicas sociales. Plantea también que la cotidianidad es una vía de incidencia directa en la estructura social.

Entendemos entonces la cotidianidad como el escenario, en tiempo y espacio donde transcurren las relaciones sociales, la construcción de creencias, categorías, costumbres se reproducen y socializan; esto implica verla como un espacio ideal para gestar transformaciones sociales y conocimientos populares. Ver la cotidianidad desde esta perspectiva nos desafía, retomando a Freire (1971) en la Pedagogía del Oprimido, a la "decodificación de lo cotidiano", es decir, a problematizar nuestras acciones, asumiendo que éstas son la semilla para la reproducción de estructura social.

Desde este horizonte, vimos la necesidad de profundizar sobre los procesos de subjetividad desde la educación popular: son los sujetos quienes forman las comunidades, los que tienen experiencias y las recrean en la construcción de conocimientos.

Los sujetos inician su reivindicación en la educación popular a través de sus experiencias vivenciales, identidades individuales, creencias, saberes y sus universos simbólicos, reconociendo la existencia de diversas lógicas y modos o métodos de producción del saber, enfatizando en la producción de sentidos de vida de manera colectiva e individual lo que permite ampliar el lenguaje y la visión al momento de expresarlo (Torres, 2000, p. 5).

Para comprender la educación popular en clave decolonial como un camino para la construcción social y cotidiana de la paz, es relevante profundizar en la manera como ocurre la educación. Esto es, revisar los conceptos de pedagogía, didáctica y mediaciones que hacen parte del proceso popular educativo. Carrillo (2007) propone que pedagogía responde al "Saber educar: qué, por qué, a quiénes y cómo se educa", y sugiere que es necesario retomar el sentido, las intenciones, los contextos y los sujetos como actores reflexivos y protagonistas de la dinámica de aprendizaje y construcción de conocimiento. 
Asumimos la didáctica como la metodología de los procesos pedagógicos, es decir, el cómo se desarrolla la dinámica entre enseñanza-aprendizaje, permitiendo el cuestionamiento de relaciones jerárquicas en la misma, mediante el uso de actividades y técnicas significativas en las que se apropian los conceptos situados en la realidad. Por lo tanto, trasciende lo instrumental facilitando que los sujetos, a través de la comunicación, creen espacios para la construcción colectiva de conocimientos y aprendizajes que propenden por la transformación, la emancipación y la libertad (...) Y son las mediaciones, los instrumentos, contenidos y formas de expresión que permiten hacer tangible la socialización del conocimiento en el marco de una educación construida desde la participación, la creatividad, la expresividad y la relacionalidad" (Arévalo, Fuentes, Hernández y Robayo, 2015, p. 32-33).

\section{Metodología}

Para construir la unidad didáctica, asumimos una metodología cualitativa con enfoque crítico de educación popular en clave decolonial, la cual nos permitió comprender cuáles eran las ideas, expectativas y preguntas frente a la paz, y no partir de supuestos. La decolonialidad dentro de la construcción del material fue un eje transversal que guió cada paso que dimos con el fin de lograr romper con el conocimiento homogeneizante $y$, por ello mismo realizamos una unidad que permitiera ser socializada con diferentes personas, contextos y momentos. Para lograrlo buscamos la participación de varios actores sociales alrededor de las nociones de paz y sobre la creación de la unidad. Desarrollamos el proceso en tres momentos como se evidencia en la tabla 1. 


\section{Tabla 1: Proceso de elaboración de la unidad didáctica}

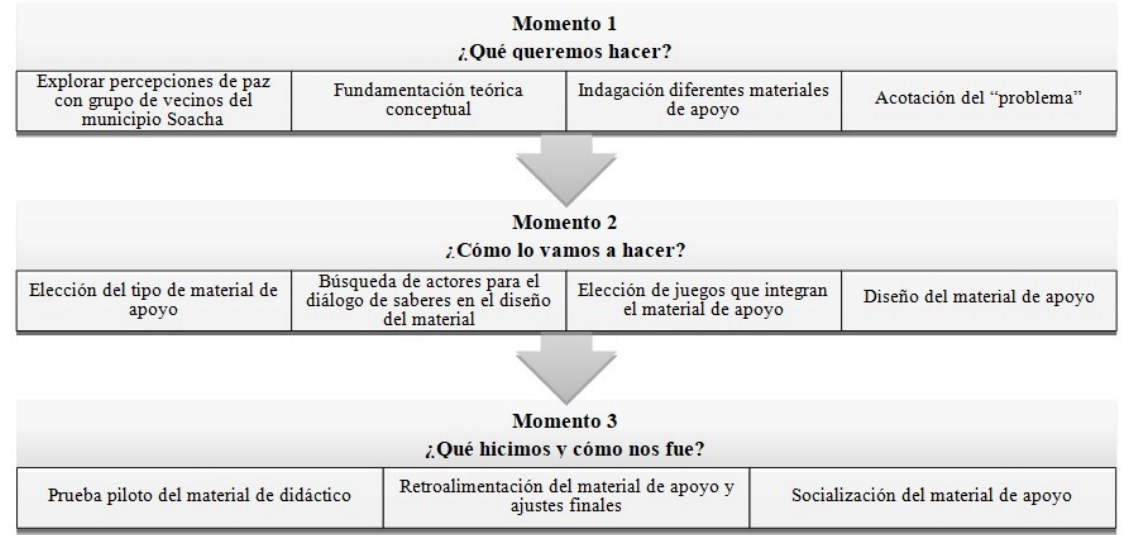

Fuente: Elaboración propia

En el primer momento, nos encontrábamos ante la incertidumbre: ¿Qué queremos hacer?, Teníamos claro que buscábamos trascender lo académico y poder llegar de manera tangible al corazón de las personas, a sus vidas, su cotidianidad, para que poco a poco, todos nos hiciéramos parte del proceso de construcción de paz; por esa razón decidimos iniciar indagando con un grupo de vecinos en el municipio de Soacha, sus percepciones acerca de la paz y del proceso que en ese momento se adelantaba en Colombia ${ }^{2}$ con preguntas orientadoras cómo: ¿Qué cree que es la paz?, ¿Qué sabe de los acuerdos de paz?, ¿Qué problemas encuentran en el barrio?, ¿Qué podrían hacer para mejorar el contexto?, ¿Cree que puede hacer algo para construir paz?.

Fruto de dicha conversación, encontramos que las personas identifican la paz como un estado en el que no hay violencia -lo que Galtung (2013)

${ }^{2}$ El trabajo de grado se desarrolla durante el año 2015, momento en el que se llevaba a cabo el tercer año de los acuerdos de paz, por medio de las mesas de diálogo con diversos sectores de la sociedad (Víctimas, líderes políticos, representación del gobierno y representación de las FARC) en La Habana, para definir los consensos sobre seis puntos para el alcance de una sociedad justa y en paz: 1. Política de desarrollo agrario integral, 2. Participación política, 3. Fin del conflicto, 4. Solución al problema de las drogas ilícitas, 5. Víctimas, 6. Implementación, refrendación y verificación. Dado el momento histórico por el que el país estaba atravesando, vimos la necesidad de hablar con la gente para conocer sus opiniones, ideas y percepciones frente a dicho proceso.

Prospectiva. Revista de Trabajo Social e intervención social No. 24, julio-diciembre 2017: pp. 177-203 
llama paz negativa- y veían el proceso de paz, como un tema netamente político, en el que solo las élites y mandatarios tenían incidencia. Sin embargo, cuando hablaban de la realidad de su barrio, hacían expresiones referentes a su papel como ciudadanos para alcanzar situaciones de justicia y resolución pacífica de los conflictos.

Este primer encuentro (Ver figura 2) nos permitió acotar el problema de investigación, es decir, la situación en la que queríamos intervenir con la propuesta de material didáctico. Luego, iniciamos la indagación conceptual con la cual fundamentamos el trabajo, lo que implicaba buscar los enfoques, perspectivas y conceptos, dentro de la epistemología decolonial y en el marco de la educación popular, y a la vez, integrar los postulados. Después de hacer la revisión de bibliografía, pudimos encontrar las conexiones y entenderlas en el marco de una espiral (como representamos en la gráfica 1 y explicamos en el apartado anterior).

Figura 2: Exploración percepciones de paz con grupo de vecinos del municipio Soacha

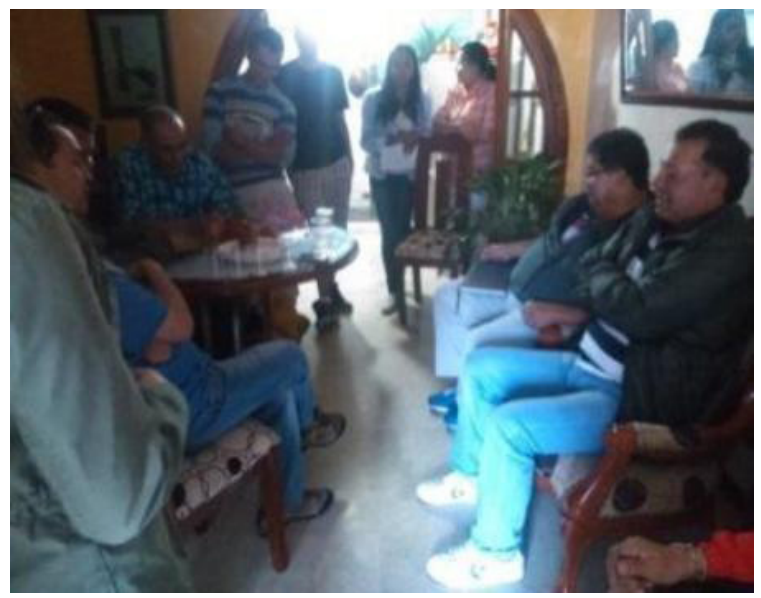

Fuente: Elaboración propia

De igual manera, hicimos una revisión sobre diferentes tipos de material didáctico que existían, sus contenidos, formas de socialización y críticas. Encontramos como materiales más comunes las cartillas, videos y juegos. Revisamos la Cartilla número 1 desarrollada por el equipo de trabajo del 
Programa por la Paz de la Compañía de Jesús, "Desarrollo de habilidades para la construcción de la paz" (Castañeda, Fernández, Acosta y Tejada, 2003). Evidenciamos que es un material que expresa conocimientos ya construidos, con premisas que responden a ideas de los autores, que pocas veces se adaptan a las realidades de las personas. Para el análisis de videos, revisamos uno realizado por la Organización Social Planeta Paz llamado ¿QUÉ ES PAZ?; y pese a ser una herramienta útil en la difusión de información, evidenciamos que se queda en la interacción audiovisual, pues no es posible desarrollar un seguimiento e identificar los efectos reales, que en términos sociales, tiene en las personas. Respecto a los juegos encontramos uno del CINEP, CHACHAFRUTO, Batalla o trueque 2002 y ¿A qué le apostamos?2007. Esta propuesta plantea reflexiones colectivas que tienen directrices específicas para el cumplimiento de los objetivos, por lo que es necesario que alguien lo dirija, para guiar al grupo a que alcance la meta propuesta.

Al identificar las potencialidades y dificultades de cada material, delimitamos lo que queríamos hacer. Siguiendo las propuestas de (Arévalo, Fuentes, Hernández y Robayo, 2015, p. 28) definimos "una unidad didáctica, como combinación creativa que puede tener variedad de formas, constituye un producto físico perdurable, que desafía el paso fugaz del tiempo, la fragilidad de la palabra y la memoria humana". Con el análisis epistemológico y de materiales, formulamos seis planteamientos que consideramos pertinentes para la construcción del material y que responden a nuestra postura ética, política y profesional. En consecuencia, esta unidad:

- Promueve el trabajo colectivo en el que las relaciones tienden a ser recíprocas y los saberes subjetivos que le otorgan sentido a las realidades son válidos.

- Busca una perspectiva crítica desde prácticas pedagógicas que pretenden transformar las realidades y propende una educación emancipadora y para la libertad.

- Es una herramienta que fomenta y fortalece la participación y la organización popular. 
- Da cuenta de los contextos, en donde se construye una planeación, una intencionalidad y una pertinencia social que es pertinente para diferentes espacios de intervención.

- Construye conocimientos a partir de procesos surgidos en los experiencial-vivencial de quienes participaron en los procesos de intervención social.

- Apoya procesos de intervención social, especialmente desde Trabajo Social

Al concretar el tipo de material y las características que debía tener, entramos en el segundo momento del proceso: logramos identificar que íbamos a construir una unidad didáctica con diferentes mediaciones, que generara recordación, y que permitiera, en coherencia con el diálogo de saberes y la educación popular en clave decolonial, reflexionar acerca de la construcción social y cotidiana de la paz.

Reflexionamos sobre las personas a las que iba dirigido el material, para quién lo hacíamos; después de varias discusiones grupales, decidimos orientar el material:

A hombres y mujeres, profesionales o no, que estén interesados en orientar procesos de reflexión acerca de la construcción social y cotidiana de la paz en sectores urbanos, en escenarios que no necesariamente son formales, de manera coherente con la apuesta de la educación popular y el diálogo de saberes. Lo anterior, ya que brindan la oportunidad de reconocer a los sujetos sociales como protagonistas de la construcción de conocimientos y cambios sociales desde la pluralidad, la particularidad, la diferencia y el trabajo colaborativo. Son los y las orientadores/as los sujetos quienes cumplen el papel de socializar el material, como una mediación pedagógica en sus procesos formativos y sociales encaminados al reconocimiento y construcción de la cotidianidad como un escenario necesario para la vivencia de la paz (Op.cit. p. 44).

El siguiente paso fue reflexionar ¿Cómo lo íbamos a hacer? Decidimos que debía ser una construcción colectiva, dar vida al diálogo de saberes y la creación de conocimientos propios. Por ello reunimos algunas personas con diferentes perfiles: amigos, familiares, compañeros de cada una de nosotras, que tenían en común "el interés por la paz”. Llegaron a nuestra 
convocatoria veinte personas, con quienes socializamos la propuesta. Juntos organizamos seis encuentros en los que buscamos recoger ideas para la construcción de las mediaciones lúdicas, juegos, y luego ensayar el fruto de la construcción colectiva, revisar las falencias y las potencialidades. (Véase Figura 3).

Figura 3. Lluvia de ideas encuentro 1.

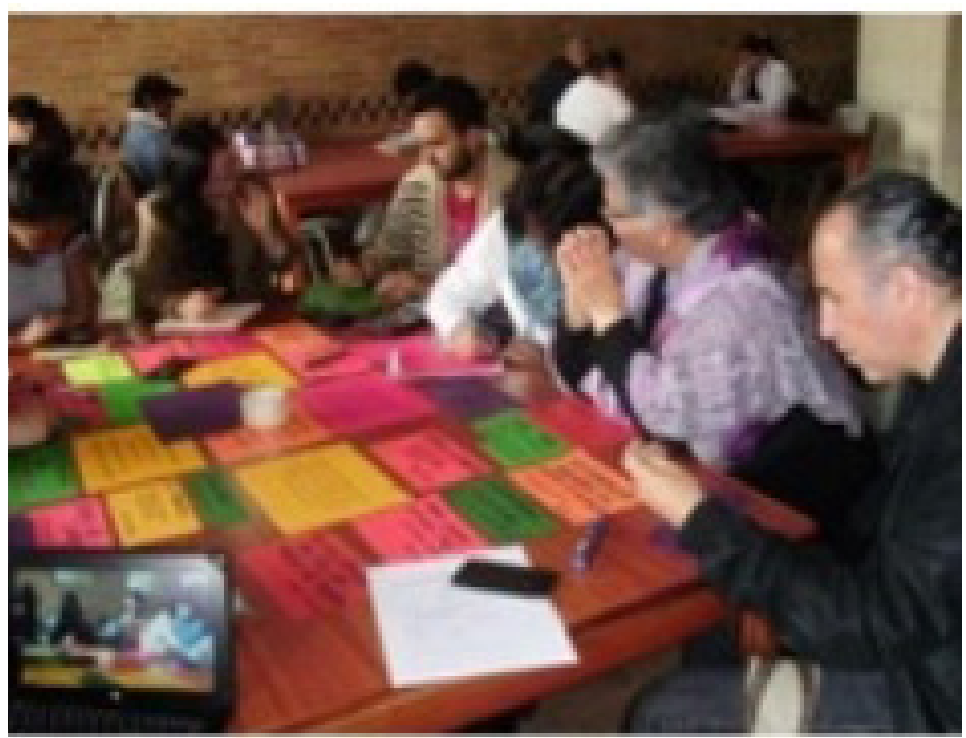

Fuente. Archivo grupal

La dinámica de encuentros, que presentamos en la tabla 2, consistía en la construcción de acuerdos previos para el desarrollo de las reuniones, la presentación del objetivo, la socialización de ideas, sugerencias, apreciaciones y conocimientos para la creación de los juegos. Finalmente, jugábamos para identificar las potencialidades y dificultades de cada juego propuesto. 


\section{Tabla 2. Planeación de encuentros para la elaboración del material}

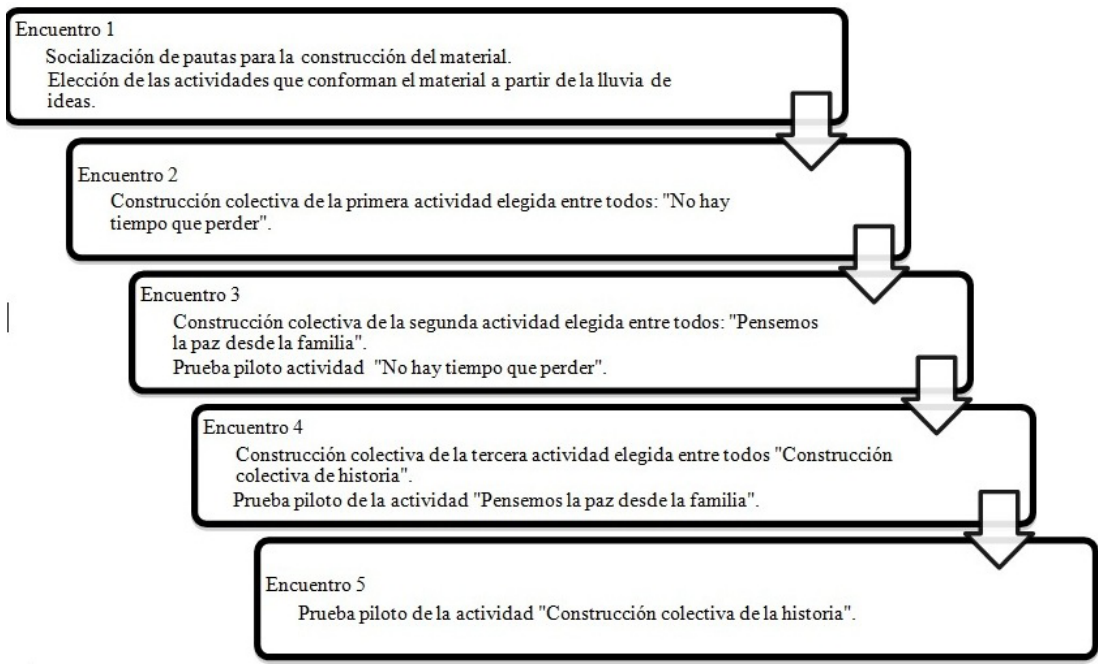

Fuente. Elaboración propia

Nuestro trabajo como grupo investigador, consistía en recoger las propuestas del encuentro y organizarlas, para dar paso a la creación de la orientación y materiales de cada juego. Socializamos dichas elaboraciones con los participantes en el siguiente encuentro, llevando a cabo "pruebas piloto" para probar cada actividad. De manera paralela, diagramamos la estructura del cuadernillo de trabajo que contenía las orientaciones de cada actividad, y diseñamos la manera cómo íbamos a presentar la unidad didáctica.

Creamos a Amy (Véase Figura 4), personaje que presenta la cartilla, como una mediación que permite construir un hilo conductor en la presentación de los juegos, y a lo largo del recorrido en el material dialoga e interactúa con los lectores. 


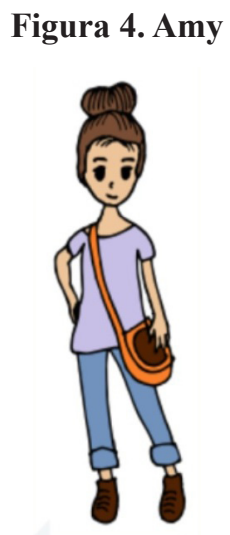

Fuente. Archivo grupal

Finalmente, realizamos un encuentro en el que socializamos toda la unidad, fruto de un trabajo participativo y colectivo, coherente con nuestras apuestas epistemológicas y metodológicas. En el último encuentro retomamos nuevas recomendaciones, que propiciaron la creación de nombres más llamativos para cada juego y la inclusión de mediaciones para complementar cada actividad. Así culminamos el momento tres del proceso, en el que vimos “¿qué hicimos? y ¿cómo nos fue?”

\section{Resultados}

Como resultado del proceso, creamos la Unidad didáctica "Vamos a construir paz todos": la nombramos de esta manera, ya que expresa nuestra intención de compartir la idea que todos hacemos parte de la construcción de paz, en coherencia con los objetivos de la investigación y con el proceso de elaboración participativa de la unidad.

La llamamos unidad didáctica, porque es un material en el que combinamos el uso de medios escritos y lúdicos con herramientas para promover escenarios de reflexión a través del juego, sobre la construcción social y cotidiana de la paz.

Enfocamos cada juego en la dimensión individual, familiar y colectiva de los sujetos, como diferentes espacios para la socialización y vivencia de 
la cotidianidad, donde en principio debemos propiciar la construcción de la paz.

La dimensión individual se relaciona con las decisiones, actitudes y valores que cada sujeto tiene y proyecta, desde la actuación propia y particular, en sus espacios relacionales; en segundo lugar, la familia como primer espacio de socialización donde se construye y fundamenta la personalidad del sujeto, donde se constituyen relaciones de afecto, que tienen un papel fundamental en la vida de cada uno de los miembros, se generan rupturas, desencuentros y conflictos. También la dimensión colectiva, relacionada con los espacios meso y macro sociales en que el sujeto se configura como ciudadano, ciudadana, amigo, amiga, trabajador, trabajadora, compañero y compañera. Es el nodo en el que los sujetos proyectan lo que son en diferentes roles, que concretan las acciones para la construcción social de la paz. Entendemos estas dimensiones en constante interrelación, por lo que la una afecta a la otra y viceversa (Véase Figura $5)$.

Figura 5. Caja de la Unidad Didáctica

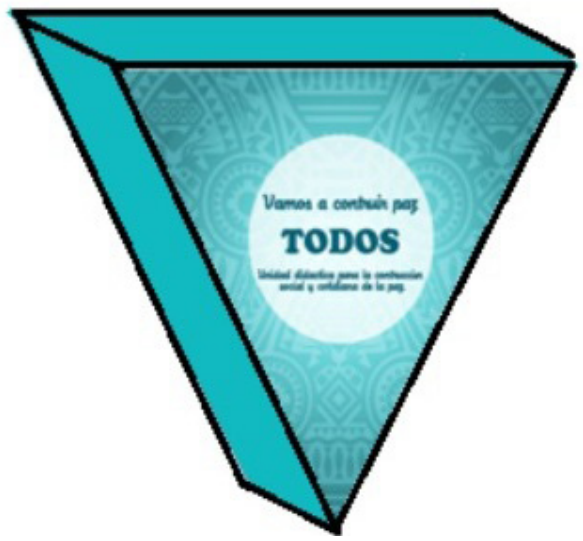

Fuente. Archivo grupal

La unidad es una caja de forma triangular: cada punta representa las tres dimensiones del sujeto y la interrelación que existe entre ellas; al abrirla se encuentra el cuadernillo de orientaciones, y tres compartimientos 
donde están los materiales de cada uno de los juegos. Amy presenta el cuadernillo, los objetivos, la población a la que está dirigida, la pertinencia social, la presentación del material y de los conceptos que fundamentaron la elaboración de la unidad, con un lenguaje cercano y comprensible para todo tipo de población.

Se presentan las orientaciones de los tres juegos planteados (Véase Figura 6): y tú, ¿qué harías?, la torre de la paz y siembra la historia, cuida el árbol de la paz, con una descripción general de cada juego, los propósitos planteados en 3 vías: conceptuales, asociadas al concepto que se pretende apropiar con el juego; procedimentales, referidas a los comportamientos que se espera lograr en el desarrollo del juego; y actitudinales, que representan las actitudes que se busca problematizar con los participantes en el desarrollo del juego.

Figura 6. Página del cuadernillo de orientaciones

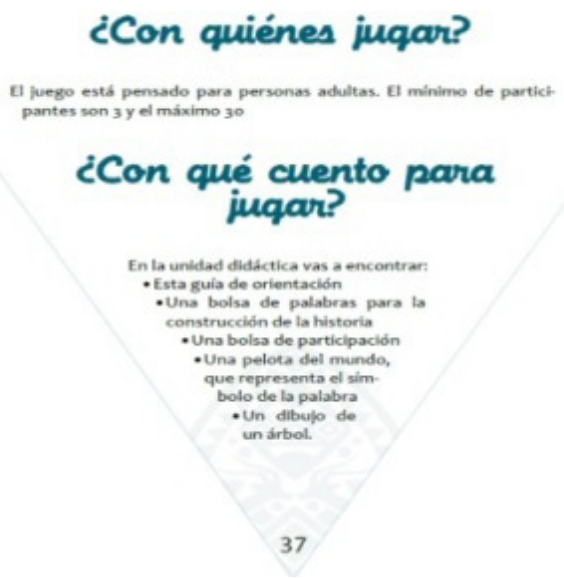

Fuente. Archivo grupal

A continuación se encuentra la respuesta a las preguntas ¿Con quiénes jugar?, ¿Con qué cuento para jugar?, y ¿Cómo juego?; en este último interrogante también se incluyen notas para tener en cuenta en la preparación y desarrollo del juego, así como preguntas para orientar la reflexión, de 
acuerdo con los objetivos del juego, las cuales buscan retomar momentos del ejercicio lúdico y compararlos con la realidad: ¿Fue fácil representar el rol asignado?, ¿Crees que tus acciones inciden en la construcción social de paz?, ¿Qué situaciones externas afectan a tu familia? ¿Qué significa tener voz en situaciones como las del juego? ¿Cuál crees que es tu papel como ciudadano en la construcción social de la paz?

El primer juego se llama "Y tú, ¿Qué harías?" (Véase Figura 7): trabaja la dimensión individual de los sujetos y busca generar reflexiones acerca de las acciones cotidianas que realizamos, y cuáles de esas contribuyen a la construcción social de la paz. Consiste en la representación de situaciones cotidianas a partir de roles específicos (natural, el deber ser y lo que no debería ser). La finalidad es que los participantes puedan ver y actuar los diferentes roles y piensen cuál de todos los identifica, con la intención que al final se reflexione alrededor de la importancia de tomar conciencia acerca de lo que hacemos diariamente, teniendo en cuenta que dichas acciones son potenciales y pueden contribuir a la construcción de la paz.

Figura 7. Juego: Y tú, ¿qué harías?

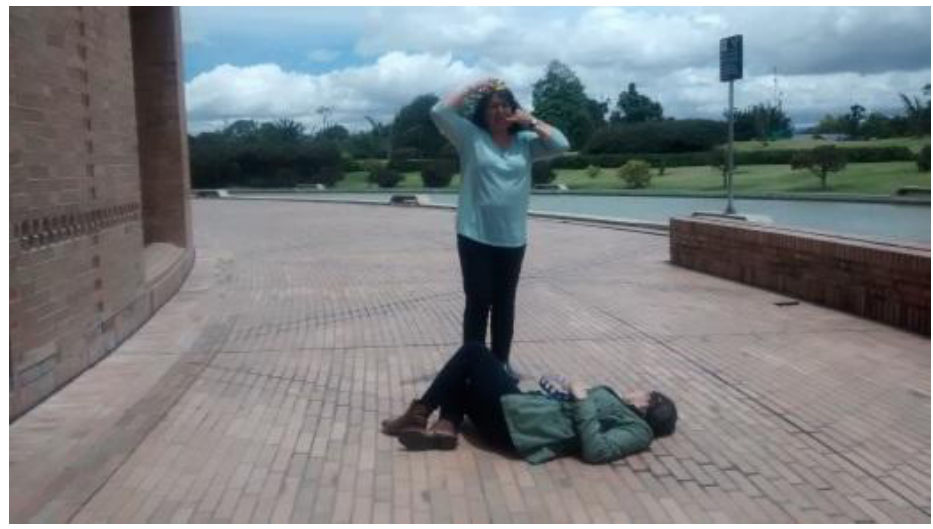

Fuente. Archivo grupal

El segundo juego es "La torre de la paz" (Véase Figura 8) que representa la dimensión familiar del sujeto; a través de la deconstrucción y posterior construcción de una torre que representa la familia, pretende generar ideas de reflexión que identifiquen la familia como un espacio primordial y como 
un sujeto potencializador de acciones dirigidas a la construcción social de la paz en la cotidianidad.

Figura 8. Juego La torre de la paz

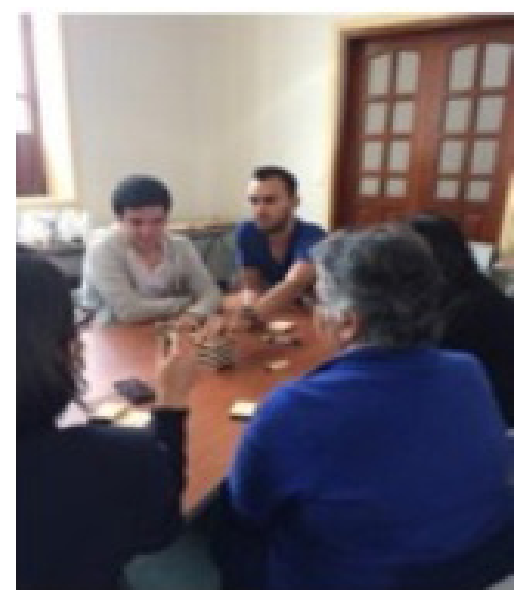

Fuente. Archivo grupal

El tercer juego se llama "Siembra la historia, cuida el árbol de paz" (Véase Figura 9): hace referencia a la dimensión colectiva del sujeto, pretende articularla con reflexiones sobre las dimensiones individual y familiar, con el fin de que los participantes discutan la interrelación de las mismas, apuntando a cómo las acciones individuales repercuten en sus escenarios familiares y colectivos, por lo que éstas deben orientarse hacia la construcción social y cotidiana de la paz. En este juego se invita a la construcción colectiva de una historia, a partir de palabras que están ubicadas en el marco de lo estructural. De igual forma, se solicita ubicarlas en un árbol que representa la manera como se construyen los conflictos y la paz, lo cual da una oportunidad para que los participantes socialicen cuál es su aporte para la construcción social de paz desde sus diferentes dimensiones, con el fin de generar conciencia acerca de la importancia del diálogo de saberes, la participación frente a escenarios que trascienden la cotidianidad pero que la condicionan, y con ello, potenciar acciones para la construcción social de paz. 
Figura 9. Juego Siembra la historia, cuida el árbol de la paz

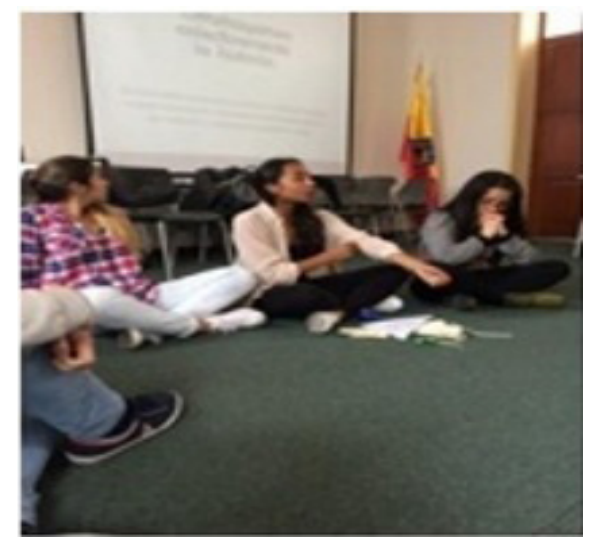

Fuente. Archivo grupal

Finalmente, se presenta un apartado nombrado "Reflexionemos", en el que se invita a los lectores y participantes a hacer un ejercicio de conciencia sobre lo que significa el accionar cotidiano como un aporte fundamental y necesario que debe potenciarse para la construcción social de la paz..

\section{Conclusiones}

La construcción de una unidad didáctica que propicie la reflexión en torno a la construcción social y cotidiana de la paz fue un proceso que tocó nuestras vidas y sentires como profesionales del Trabajo Social, también como ciudadanas, mujeres, hijas de la historia que se ha escrito en Colombia.

A lo largo de la elaboración del material logramos identificar que aún se encuentran muchos caminos sin andar, puertas sin abrir y corazones por sensibilizar para que se sumen, con pequeñas acciones, a la posibilidad de transformar nuestra realidad y paso a paso hacer la paz.

Como trabajadoras sociales, evidenciamos que desde nuestra profesión no se han desarrollado materiales como el que creamos; tampoco se ha estudiado de manera profunda la decolonialidad, como una perspectiva coherente con las intenciones transformadoras de América Latina, y 
como una posibilidad de crear nuestros conocimientos, entender nuestras problemáticas y solucionarlas con las realidades que nos permean, y no con los caminos sugeridos desde "el norte".

Desde la academia, procesos que implican investigación y producciónsocialización, no son apoyados totalmente, es decir, aunque la misionalidad de las instituciones "promueve la innovación social", en el momento en el que los estudiantes asumen el reto, se presentan varias dificultades en los lineamientos, tiempos de evaluación, desarrollo de los procesos investigativos, facilitación de espacios y recursos para la socialización. Se tratan de manera genérica las producciones, sin aportar a la potenciación de las elaboraciones de los y las estudiantes. Encontramos que no hay suficiente claridad en la orientación de procesos innovadores; por ello se quedan en las bibliotecas y repositorios institucionales impidiendo que, desde la universidad, se propenda por trascender las paredes que nos alejan de la sociedad en la que queremos incidir. Esto visto desde las barreras que pone la academia para el ingreso de personas externas a escenarios de investigación y participación; la no generación de espacios de socialización en los campos de práctica, en las clases, en los estudiantes e incluso en el resto de la universidad: el conocimiento construido por el Programa de Trabajo Social se queda ahí, no sale a las facultades, no llega a los decanos ni a los estudiantes de otras disciplinas.

La universidad no está preparada para la decolonización de conocimientos porque sigue basada en los principios de educación bancaria de los que hablaba Freire, sigue llenando salones, pero no cerebros ni corazones con ideas de transformación social, de construcción de conocimientos, de socialización sin relaciones verticales; sino que crea profesionales que repiten lo que está dicho, que no cuestionan la realidad y que no aportan a la construcción de conocimiento.

Comprendimos que la paz es un proceso social, colectivo y cotidiano, que requiere la conciencia y crítica de nosotros como ciudadanos y sujetos frente al mundo en el que estamos, para que sea un ejercicio sostenible. Esto implica reconocer nuestros saberes, crear nuestros conocimientos y promover escenarios de encuentro para el diálogo de saberes, la aceptación y potenciación de la diferencia; y la transformación de relaciones violentas 
en interlocuciones respetuosas, pacíficas y armoniosas. No se trata de pensar utópicamente un mundo sin conflictos, sino de aprender a tramitarlos de maneras creativas para solucionarlos sin agredir al otro.

Con base en esto, entendemos la educación como un ejercicio necesario y fundamental de repensarse desde lo popular, con relaciones horizontales y fuera de las ideas hegemónicas y bancarias. Por el contrario como un hacer socializante e inacabado, en el que los protagonistas son los sujetos y sus realidades, experiencias, vivencias que se encuentran en la cotidianidad, espacio-lugar en donde se vive la estructura social, y se perpetúa o transforma, por ello es en lo cotidiano, en donde podemos empezar a transformar el mundo, para participar e incidir en las estructuras, para dejar de lado la indiferencia y el individualismo, para construir el conocimiento y para cambiar la historia de nuestro país.

Los retos como profesionales, sujetos ciudadanos están dirigidos a potencializar el ejercicio didáctico como una manera alternativa y creativa de sentir-pensar, de actuar y transformar. De igual forma, esperamos tener las fuerzas para continuar compartiendo la unidad con más personas, comunidades y colectivos, de seguir soñando con realidades decolonizadas y de aportar a la construcción de una sociedad más justa y respetuosa con los demás y con la naturaleza. También nos retamos desde el Trabajo Social, para que la profesión sea uno de los escenarios que continúen promoviendo la necesidad de reflexionar, crear y compartir ejercicios innovadores que apunten a la construcción social y cotidiana de la paz, ya sea desde la academia, desde nuestro quehacer y sobre todo desde nuestro día a día.

Encontramos que nuestro material es fruto de una reflexión sentida y pensada en la que buscamos socializar la idea de que la paz también podemos construirla desde la cotidianidad, transformando prácticas y actitudes, generando reflexiones acerca de la decolonialidad como una ruptura con lo hegemónico, con lo homogeneizante, con lo establecido; reivindicando la diferencia como oportunidades para construir paz y sociedad con valores de solidaridad, reciprocidad y amor.

Finalmente, creemos que todas las reflexiones que desde la sociedad civil podamos adelantar, resultan pertinentes en el marco del proceso que ha vivido el país, de su historia, de sus muertos, de sus vivos y de las 
luchas que seguimos gestando para construir una sociedad más justa, con condiciones y políticas sociales que beneficien al conjunto y no a sectores específicos, con actitudes que desde cada sujeto permitan la interrelación pacífica, que acepten la diferencia en el pensamiento y la potencien en el bienestar común, con estrategias para afrontar los conflictos como oportunidades creativas para crecer. Sugerimos continuar pensando de manera decolonial, educando desde lo popular y dialogando con los saberes cotidianos y contextuales de cada sujeto y ciudadano del mundo, como un camino que es construido y caminado por nosotros mismos, por los colombianos, por los acallados de la colonización, por los excluidos y por los explotados. Esperamos que el actual proceso de diálogo de paz prospere, pero más allá de eso, que asumamos el compromiso de tomar conciencia de nuestras actitudes y comportamientos, y de transformarlos para la construcción social y cotidiana de la paz.

\section{Referencias bibliográficas}

Arévalo, C.; Fuentes, A.; Hernández, A. y Robayo, M. (2015). Unidad Didáctica: Vamos a construir paz todos. Un aporte desde el diálogo de saberes a la construcción social de la paz. (Tesis de pregrado). Universidad de la Salle. Facultad de Ciencias Económicas y Sociales. Trabajo Social. Bogotá, Colombia.

Bautista, C. (2013). La paz como construcción social: Papel de la participación social y popular. En J. Estrada Álvarez, Solución política y proceso de paz en Colombia. A propósito de los diálogos entre el gobierno y las FARC - EP (pp. 171-196). Bogotá: Ocean Sur.

Castañeda, R.; Fernández, C.; Acosta, A. y Tejada, C. (2003). Proceso de formación. Desarrollo de habilidades para la construcción de la paz. Cartilla No. 1. Colombia: Programa por la paz / Compañía de Jesús. Recuperado de http://tropenbos.sena.edu.co/DOCUMENTOS/MATERIALES\%20DE\%20 REFERENCIA/2\%20propaz_habipaz.pdf

CINEP. (2007). A qué le apostamos. Bogotá: Programa por la paz.

De Pablos, J. C.; Gómez, Y. y Pascual, N. (1999). El dominio sobre lo cotidiano: la búsqueda de la calidad de vida. Reis: Revista Española de Investigaciones Sociológicas, 86, 55-78. Recuperado de http://www.jstor.org/stable/40184145

De Sousa, B. (2010). Una epistemología del Sur. En De Sousa, B. Refundación del Estado en América Latina. Perspectivas desde una epistemología del 
Sur (pp. 27-71). Bogotá: Siglo del hombre editores. Recuperado de http:// www.boaventuradesousasantos.pt/media/Refundacion\%20del\%20Estado_ Lima2010.pdf

Dussán, M. A. (2004). Modelo pedagógico de las experiencias de educación popular de la Universidad Surcolombiana. (Tesis de doctorado). Universidad Autónoma de Barcelona. Facultad de Ciencias de la Educación. Barcelona, España. Recuperado de http://www.tdx.cat/bitstream/handle/10803/5282/ madc1de1.pdf

Escola de Cultura de Pau. (2011). Educar para una cultura de paz. Quaderns de construcció de Pau, 20, 4-8. Recuperado de http://escolapau.uab.es/img/qcp/ educar_cultura_paz.pdf

Fontan, V. (2013). Descolonización de la Paz. Cali: Universidad Javeriana de Cali. Freire, P. (1964). Cambio. Buenos Aires: Editorial América Latina.

Freire, P. (1966). Pedagogía de la esperanza. México: Siglo XXI.

Freire, P. (1971). Conciencia crítica y liberación: "Pedagogía del oprimido". Bogotá: América Latina.

Galtung, J. (2003). Violencia cultural. Guernika-Luno: Gernika Gogoratuz.

Gárcia-Durán, M. (2006). Movimiento por la paz en Colombia 1978-2003. Bogotá: Ediciones Antropos Ltda.

Heller, Á. (1987). Sociología de la vida cotidiana. Barcelona: Ediciones Península. Marañon, B. (2016). Notas sobre la solidaridad económica y la descolonialidad del poder En B. Marañon, Políticas para la solidaridad económica y el buen vivir en México (pp. 13-52). Ciudad de México: Universidad Nacional Autónoma de México.

Mignolo, W. D. (2007). El pensamiento decolonial: desprendimiento y apertura. En S. Castro-Gomez, El giro decolonial: reflexiones para la diversidad epistémica más allá del capitalismo global (pp. 25-46). Bogotá: Siglo del Hombre Editores.

Quijano, A. (1992). Colonialidad y modernidad/racionalidad. Perú Indígena, 13(29), 11-20. Recuperado de https://problematicasculturales.files.wordpress. com/2015/04/quijano-colonialidad-y-modernidad-racionalidad.pdf

Torres, A. (2000). Sujetos y subjetividad en la educación, Educación popular, subjetividad y sujetos sociales. Pedagogía y Saberes, 15. Recuperado de http:// www.pedagogica.edu.co/storage/ps/articulos/pedysab15_04arti.pdf

Cómo citar este artículo:

Fuentes-Oliveros, A. L. y Arévalo-Olaya, F. C. (2017). Vamos a construir Paz todos: Unidad didáctica para la reflexión acerca de la construcción social y cotidiana de la paz. Prospectiva, 24, 177-203. Doi: 10.25100/ prts.v\%vi\%i.4544 